\title{
IMMERSIONS AND EMBEDDINGS OF TOTALLY GEODESIC SURFACES
}

\author{
D. D. LONG
}

The work of Waldhausen, Thurston and others has shown that the existence of an embedding of a closed, orientable, incompressible surface in a 3-manifold is a great help in the understanding of that manifold. Unfortunately many examples exist of manifolds which contain no such embedding. However, it does seem at least conjecturally possible that any irreducible manifold with infinite fundamental group could contain an immersion of such a surface, and this has motivated the study of the question of whether such a surface can always be lifted to an embedding in some finite covering of the 3-manifold. The general question seems to be some way from resolution; the purpose of this note is to give an affirmative answer in a very special case.

THEOREM 1. Let $M$ be a closed, hyperbolic 3-manifold which contains a totally geodesic immersion of a closed surface. Then:

(a) there is a finite covering of $M$ which contains an embedded closed, orientable, totally geodesic surface;

(b) there is a finite covering of $M$ which contains an embedded non-separating, closed, orientable, totally geodesic surface.

The restriction to the closed case is unnecessary, and is made only to save some words. Standard results and terminology of hyperbolic geometry will be used without proof; good references can be found in [3] or [5].

By a slight adaptation of the technique used to prove Theorem 1, we are also able to show the following.

THEOREM 2. Let $G$ be the fundamental group of a closed, hyperbolic 3-manifold. Then the cyclic subgroups of $G$ are virtually separable.

The traditional group-theoretic condition used to deal with immersion to embedding problems is subgroup separability or LERF. We now define this despite the fact we shall use a somewhat weaker property.

If $G$ is a group and $H \leqslant G$, let us define $H^{*}=\bigcap\{K \mid H \leqslant K \leqslant G$ and $K$ has finite index in $G$ \}. Then $G$ is said to be LERF if for each finitely generated $H \leqslant G$ we have $H=H^{*}$.

The reason for introducing the notation $H^{*}$ is that such a strong condition as LERF is often not necessary; for many purposes the knowledge that the index [ $\left.H^{*}: H\right]$ is finite suffices. In [2], this condition was called virtual separability.

Received 13 October 1986.

1980 Mathematics Subject Classification 57N10, 51M10.

Bull. London Math. Soc. 19 (1987) 481-484 
If $G$ is a discrete torsion-free subgroup of $\operatorname{PSL}_{2}(\mathbb{C})$, then we shall use the symbol $\Lambda G$ to mean the limit set of $G$, that is, the closure in the sphere at infinity of the accumulation points of any-orbit. Then our main algebraic result is as follows.

LEMMA 1. Let $G$ be the fundamental group of a closed, hyperbolic 3-manifold and $H<G$ a fuchsian subgroup. Then $\Lambda H^{*}=\Lambda H$.

If for the time being we assume this result, we may prove the theorems.

Proof of Theorem 1(a). Since the lift of a totally geodesic surface continues to be totally geodesic, by passing to a double covering if necessary, we may suppose that $M$ is orientable.

Let $i: F \rightarrow M$ be a totally geodesic immersion of a closed surface. Let $G$ be a discrete faithful representation of the fundamental group of $M$ into $\mathrm{PSL}_{2}(\mathbb{C})$ and $H=i_{*} \pi_{1}(F)$. Then $H$ is fuchsian. By the Lemma, $\Lambda H=\Lambda H^{*}$, so that $H^{*}$ is also fuchsian. In fact, if we use the symbol $C(\Lambda H)$ for the convex hull of $\Lambda H$ (that is, the intersection of all the hyperbolic half spaces which contain $\Lambda H$ ) this is a hyperbolic plane $\mathbb{H}^{2}$ which is preserved by both $H$ and $H^{*}$. The action of both groups is torsion free and properly discontinuous, so if we set $F^{\prime}$ to be $\mathbb{H}^{2} / H^{*}$, this is a surface and the induced map $F \rightarrow F^{\prime}$ is a covering map. Since the surface $F$ is a finite area hyperbolic surface, so is $F^{\prime}$. It follows that the index $\left[H^{*}: H\right]$ is finite; it is in fact the area ratio: $\operatorname{Area}(F) / \operatorname{Area}\left(F^{\prime}\right)$. Thus we have exhibited the subgroup $H$ as virtually separable in $G$.

It is easy to see that in our case $\left(H^{*}\right)^{*}=H^{*}$, so that the surface group $H^{*}$ is genuinely separable in $G$, and it follows by a standard argument [4] that there is a finite covering of $M$, say $M_{f}$, to which the surface $F^{\prime}$ lifts as an embedding and for which the inclusion $F^{\prime} \rightarrow M_{f}$ realises the inclusion $H^{*}=i_{*} \pi_{1}\left(F^{\prime}\right) \rightarrow \pi_{1}\left(M_{f}\right)$.

The proof of the theorem will be complete when we have obtained an orientable surface. We do this by observing that if the surface $F^{\prime}$ were not orientable, there would be a fuchsian subgroup of index two in $H^{*}$ corresponding to the orientable double covering $F^{\prime \prime}$ of $F^{\prime}$. We can construct a double covering of $M_{f}$ by taking two copies of the manifold $M_{f} \backslash F^{\prime}$ and doubling, to obtain a covering of $M$ in which the totally geodesic surface $F^{\prime \prime}$ embeds.

RemarK. Notice that the proof does not proceed by lifting the given embedding.

Proof of Theorem 1(b). As in Theorem 1(a), we let $H$ be the fuchsian subgroup corresponding to the embedded, orientable totally geodesic surface $F$, and as in Theorem 1(a), we must have that $H=H^{*}$.

Let the two components of $M \backslash N(F)$ be denoted $C_{ \pm}$. Then it is easy to see that both of the inclusions $\pi_{1}(F)=H \rightarrow \pi_{1}\left(C_{ \pm}\right)$exhibit $H$ as a subgroup of infinite index. So we may choose $g_{ \pm} \in C_{ \pm}$, so that no power of $g_{ \pm}$lies in $H$. Then since $H=H^{*}$, it follows that there is a subgroup $K$, of finite index in $\pi_{1}(M)$, which contains $H$ but fails to contain either of $g_{ \pm}$. Let $N$ be the intersection of all the $\pi_{1}(M)$ conjugates of $K$. It is now easy to see that every lift of $F$ into the covering corresponding to the subgroup $N$ must be non-separating.

Proof of Lemma 1. By conjugating $G$ if necessary, we may suppose that the group $H$ lies inside $\operatorname{PSL}_{2}(\mathbb{R})$. Suppose that $G$ is generated by the matrices $g_{1}, \ldots, g_{t}$. If we 
denote complex conjugation by $\tau$, let $R$ be the subring of $\mathbb{C}$ generated by all the entries of the matrices $g_{i}$, all the complex conjugates of these entries, together with 1 . Then $R$ is a finitely generated integral domain with a 1 and we have that $G$ and $\tau G$ both embed in the group $\operatorname{PSL}_{2}(R) \leqslant \operatorname{PSL}_{2}(\mathbb{C})$. Recall that the symbol $J(R)$ in ring theory is used to denote the intersection of all the maximal ideals of $R$. We need the following result (see [1] or [6]).

LemMa 2. Let $R$ be a finitely generated integral domain with a 1. Then:

(a) $J(R)=(0)$;

(b) if $\mathfrak{M}$ is a maximal ideal of $R$, then the quotient $R / \mathfrak{M}$ is a finite field.

In order to prove the result of Lemma 1, it suffices to show that given any element $g$ of $G$ which has at least one of its two fixed points off $\Lambda H$, there is a homomorphism of $G$ onto a finite group for which the image of $g$ does not lie in the image of $H$. Since we have normalised so that $\Lambda H=\mathbb{R}$, any such $g$ must have a non-real entry in its matrix. Call this entry $x$. Then $x-\tau x$ is non-zero, so by Lemma 2(a) there is a maximal ideal $\mathfrak{M}<R$ which does not contain this element. Let $\pi: R \rightarrow R / \mathfrak{M}$ denote the projection map. Then we have a homomorphism $\rho: \operatorname{PSL}_{2}(R) \rightarrow \operatorname{PSL}_{2}(R / \mathfrak{M}) \times \operatorname{PSL}_{2}(R / \mathfrak{M})$ defined by sending the matrix $\gamma$ to $(\pi(\gamma), \pi \tau(\gamma))$. The right-hand side of this equation is a finite group and by restriction we obtain a map $\rho: G \rightarrow \operatorname{PSL}_{2}(R / \mathfrak{M}) \times \operatorname{PSL}_{2}(R / \mathfrak{M})$. Observe that the image of the element $g$ is a pair of matrices which are distinct, since they differ in the place corresponding to $x$. On the other hand all the matrices of $H$ map in as identical pairs, since all their entries are real. Hence $\rho g$ cannot lie in $\rho H$ and the Lemma is proven.

The above result has been proved independently by $\mathrm{G}$. Mess.

We now embark on the proof of Theorem 2 . This entails a slight variation on the above theme.

Proof of Theorem 2. Recall that we can think of the isometry group of hyperbolic 3 -space as $\mathrm{SO}^{+}(3,1)$. Then given a hyperbolic 3-manifold, its fundamental group $G$ admits a discrete faithful representation into $\operatorname{SO}^{+}(3,1)$ and hence via the natural inclusion, into $\mathrm{SO}^{+}(4,1)$. Call this totally geodesic representation $\rho: G \rightarrow \mathrm{SO}^{+}(4,1)$. If we identify hyperbolic 4-space with the interior of the 4-ball, we can think of the limit set of $G$ as being contained inside a 'level' $B^{3} \subset B^{4}$.

Fix some element $\gamma \in G$, and recall that we seek to show that $\left[\langle\gamma\rangle^{*}:\langle\gamma\rangle\right]\langle\infty$. Choose any $h \in G$ whose fixed points do not coincide with those of $\gamma$. There is an element $\Gamma \in \mathrm{SO}^{+}(4,1)$ whose axis is equal to that of $\gamma$ and whose action on the element $h$ is to move at least one of its fixed points off the 'level' $B^{3}$. Using the element $\Gamma$, we can conjugate the representation $\rho$, to obtain a new representation $\rho^{\Gamma}: G \rightarrow \mathrm{SO}^{+}(4,1)$, whose limit set has been 'twisted', so that the fixed points of $\gamma$ still lie in the level $B^{3}$, and at least one of the fixed points of $h$ does not.

There is an involution $\tau$ of $\mathbb{T}^{4}$ which reflects in the level $B^{3}$. Then $\tau$ commutes with $\gamma$, so leaves its matrix in $\mathrm{SO}^{+}(4,1)$ unchanged, but $\tau$ definitely changes the matrix of $h$. Now we may proceed exactly as in the proof of Lemma 1 , using the map $\tau$ in place of complex conjugation, to find a finite representation of $G$ for which the image of $h$ avoids the image of the subgroup $\langle\gamma\rangle$. 
It follows that the only elements which cannot be so separated from $\gamma$ are those which have the same fixed points as $\gamma$; whence $\left[\langle\gamma\rangle^{*}:\langle\gamma\rangle\right]\langle\infty$, as required.

COROLLARY 1. If $G$ is the fundamental group of a hyperbolic 3-manifold which is a surface bundle over the circle, the cyclic subgroups are genuinely separable.

Proof. Surface groups are LERF (see [4]), and the corollary is an easy consequence of this fact and Theorem 2.

COROLlaRY 2. A primitive geodesic in a closed hyperbolic 3-manifold may be lifted to a simple geodesic in some finite covering.

RemarK. A topological argument shows that every loop on a surface can be lifted to a primitive loop in some finite regular covering; so we deduce that for fuchsian groups, the cyclic subgroups are genuinely separable. This, of course, is contained in [4].

Postscript. Since proving the result of Theorem 2, it was brought (rather indirectly) to the author's attention that a more general theorem was already known to algebraists, namely:

Proposition. Let $G$ be a residually finite group. Then if $A$ is a maximal abelian subgroup in $G, A=A^{*}$.

In the context of closed, hyperbolic 3-manifold groups, the maximal abelian subgroups are cyclic, so Theorem 2 follows. In fact, the proof of the Proposition shows a little more; namely that a subgroup in a residually finite group which is maximal subject to some verbal law is separable.

Proof of Proposition. Let $f\left(X_{1}, \ldots, X_{n}\right)$ be some abstract polynomial, and let $H$ be a subgroup which is maximal subject to $f\left(h_{1}, \ldots, h_{n}\right)=1$ for all $h_{i} \in H$. (In the abelian case we choose $f\left(X_{1}, X_{2}\right)=X_{1} X_{2} X_{1}^{-1} X_{2}^{-1}$.) Since $G$ is residually finite, we can choose a sequence of normal subgroups of finite index $N_{i}$, with $\bigcap N_{i}=\{e\}$. Now observe that for each $N_{i}$ we have $f\left(h_{1} N_{i}, \ldots, h_{n} N_{i}\right) \in N_{i}$, since this projects trivially into $G / N_{i}$. Hence $\bigcap f\left(H N_{i}, \ldots, H N_{i}\right)=\{e\}$, so that the subgroup $\bigcap H N_{i}=H^{*}$ satisfies $f$. Since $H$ was chosen to be maximal for this, it follows that $H=H^{*}$.

\section{References}

1. I. KAPLANSKY, Commutative rings (Allyn and Bacon, 1970).

2. D. D. LONG, 'Engulfing and subgroup separability for hyperbolic groups', preprint.

3. J. Morgan and H. Bass (editors), The Smith conjecture, Proc. Conf. Columbia (Academic Press, 1984).

4. G. P. Scotr, 'Subgroups of surface groups are almost geometric', J. London Math. Soc. 17 (1978) 555-565.

5. W. P. Thurston, 'The geometry and topology of 3-manifolds', Princeton lecture notes.

6. B. A. F. Wehrfritz, Infinite linear groups (Springer, Berlin, 1973).

Department of Mathematics

University of California

Santa Barbara, CA 93106, USA 